\title{
CATÓlICOS, EVANGÉLICOS Y DERECHOS HUMANOS: UN ESTUDIO DE SUS DIFERENCIAS EN ESTUDIANTES SECUNDARIOS CHILENOS ${ }^{1}$
}

\author{
RODOLFO NÚÑEZ HERNÁNDEZ* \\ CECILIA RAMÍREZ VENEGAS** \\ KRETY SANHUEZA VIDAL $* * *$ \\ NILDA QUEZADA MAYA****
}

\begin{abstract}
RESUMEN
La investigación examina la relación entre religión y derechos humanos de estudiantes chilenos de educación secundaria y se observa esta relación para estudiantes católicos y evangélicos.

Los esquemas teóricos acerca de la construcción de valores y su relación con la apreciación de determinados derechos humanos describen la adquisición de pautas de conducta que permanecen en el tiempo, producto de la participación de agencias de socialización como la familia y la escuela.

El ethos religioso de estudiantes cristianos, utilizados en este estudio cuantitativo con técnicas de correlación y regresión múltiple, muestra la posición significativamente contraria a la eutanasia y la pena de muerte y una disposición positiva frente a la regulación de instituciones externas, como el Estado, en la práctica de la sexualidad en contexto privado. Para católicos y evangélicos las diferencias son pequeñas, pero muestran posiciones diferentes que se explican por aspectos teóricos de conformación de ambas religiones en Chile, su condición de mayoría o minoría en el contexto de la agencia escuela y la «anomia política».

A medida que los cristianos, o en su distinción entre católicos y evangélicos, se alejan de su práctica y compromiso con su religión, las respuestas se hacen menos significativas o definitivamente opuestas.
\end{abstract}

PALABRAS CLAVE: DERECHOS HUMANOS, RELIGIÓN, EDUCACIÓN, CATÓLICOS, EVANGÉLICOS

1 Esta investigación se enmarca en el proyecto de investigación del equipo de Religión, educación y derechos humanos, del Centro de Estudios de la Religión, de la UC.

* Doctor en Psicología, Facultad de Educación, Psicología y Familia, Universidad Finis Terrae, Santiago, Chile. Correo electrónico: rnunez@uft.cl

** Doctora en Ciencias de la Educación, Facultad de Educación, Pontificia Universidad Católica de Chile, Santiago, Chile. Correo electrónico: dramirev@uc.cl

*** Doctora en Teología, Instituto de Ciencias Religiosas, Pontificia Universidad Católica de Valparaíso, Valparaíso, Chile. Correo electrónico: krety.sanhueza@pucv.cl

**** Socióloga. Magíster en Sociología. Correo electrónico: nilda@coiro.cl 


\title{
CATÓLICOS, EVANGÉLICOS E DIREITOS HUMANOS: UM ESTUDO DE SUAS DIFERENÇAS EM ESTUDANTES CHILENOS DO ENSINO MÉDIO
}

\section{RESUMO}

A pesquisa examina a relação entre a religião e os Direitos Humanos de estudantes chilenos do ensino médio sendo observada esta relação em estudantes católicos e evangélicos.

Os esquemas teóricos sobre a construção de valores e sua relação com a apreciação de certos direitos humanos, descrevem a aquisição de padrões de comportamento que permanecem ao longo do tempo como resultado da participação de agências de socialização como a família e a escola.

O ethos religioso dos estudantes cristãos, utilizados neste estudo quantitativo com técnicas de correlação e regressão múltipla, mostra a posição significativamente contrária à eutanásia e à pena de morte e uma disposição positiva em relação à regulação de instituições externas, como o Estado, na prática da sexualidade no contexto privado. Para católicos e evangélicos as diferenças são pequenas, mas mostram posições diferentes que são explicadas por aspectos teóricos de conformação de ambas as religiões no Chile, sua condição de maioria ou minoria no contexto da agência escola e a "anomia política".

À medida que os cristãos, ou em sua distinção entre católicos e evangélicos, afastam-se da sua prática e compromisso com sua religião, as respostas se tornam menos significativas ou definitivamente opostas.

PALAVRAS-CHAVE: DIREITOS HUMANOS, RELIGIÃO, EDUCAÇÃO, CATÓLICOS, EVANGÉLICOS

\section{CATHOLICS, EVANGELICALS AND HUMAN RIGHTS: A STUDY ON THEIR DIFFERENCES IN CHILEAN SECONDARY SCHOOL STUDENTS}

\begin{abstract}
This research examines the relation between religion and Human Rights in Chilean Catholic and Evangelical secondary school students.

The theoretical schemata about the construction of values and their relation with the appreciation of certain human rights, describe the acquisition of behavioral patterns that remain through time as a result of the involvement of agencies of socialization such as family and school.

The religious ethos of these Christian students, measured in this quantitative research through correlation and multiple regression analysis, shows minimal differences between Catholics and Evangelicals in their value base; their position is significantly contrary towards euthanasia and death penalty, and significantly supportive towards the regulation of external institutions such as the State, concerning to the practice of sexuality in private contexts. Differences between Catholics and Evangelicals can be still evidenced, nevertheless, by the theoretical aspects of both religions in Chile, their minority or majority condition in the context of the school, as an agency of socialization, and "political anomie". As Christians, in their differentiation between Catholics and Evangelicals, drift away from their practice and commitment with their own religion, answers will become either less significant or completely opposite.
\end{abstract}

KEYWORDS: HUMAN RIGHTS, RELIGION, EDUCATION, CATHOLICS, EVANGELICALS 


\section{INTRODUCCIÓN}

Esta investigación se origina en un esfuerzo por avanzar y profundizar, en el trabajo realizado por Hans-Georg Ziebertz y Marion Reindl, en el marco del programa internacional empírico sobre religión y derechos humanos $2.0 \mathrm{y}$ que lleva como nombre: «Socialización religiosa y sus valores como predictores de las actitudes de los derechos humanos: Un estudio empírico entre adolescentes cristianos y musulmanes en Alemania. (2011-2017)».

Dicho estudio se llevó a cabo adaptando la encuesta original al medio nacional $^{2}$. En Chile se aplicó en nueve ciudades, a estudiantes de tercero y cuarto de enseñanza media o secundaria, de colegios laicos, protestantes y católicos.

Para este estudio y dada la historia de la constitución del cuerpo de creencias religiosas en Chile, el espectro de sensibilidades de la fe recupera fundamentalmente a creyentes de matriz cristiana, esto hace que la cantidad de musulmanes - estrato que se ocupó en el estudio original- carezca de significación estadística; esto, a diferencia de lo que ocurre con la investigación, que es la base de la aplicación en Chile. En este estudio, nos interesó tomar de la muestra a los estudiantes cristianos y, a este conjunto, estratificarlo por católicos y evangélicos ${ }^{3}$, para observar eventuales diferencias en torno a temas de derechos humanos. En cada uno de estos grupos se consideró como variables independientes los aspectos referidos a: nivel de ritualidad: rezar; asistencia a celebraciones; nivel de pluralismo religioso: equivalencia de la religión; ausencia de diferencia entre religiones; nivel de validación religiosa: exclusivismo religioso; religión ejerce influencia en la vida.

Estas variables fueron consideradas para establecer la diferencia en la gradualidad de la fuerza que puede ejercer en un individuo su ethos religioso.

Desde lo antes planteado, se buscó establecer correlaciones y regresiones múltiples, con ciertas variables de DD.HH., en las que se hipotetizaron diferencias, que están en el plano de la sexualidad, la acción pública y el derecho a la vida ${ }^{4}$. Las variables fueron identificadas como: derechos a privacidad: privacidad sexual sin intervención del Estado (derecho civil); derecho a protestar: garantía de la protesta a pesar de riesgo en orden público; garantía de la protesta pese a ser cuestionable su demanda; derecho a la vida: contra eutanasia; contra pena de muerte.

\footnotetext{
${ }^{2}$ Manzi y Silva (2014).

${ }^{3}$ Las diversas sensibilidades evangélicas no alcanzan un tamaño muestral adecuado para ser tratadas en este estudio. Razón que hace que sean consideradas como un estrato único.

${ }^{4}$ Para la variable dependiente del derecho a la vida no se consideró la opinión respecto del aborto, ya que en paralelo a esta investigación se realizó un estudio, de similares características, que tomó dicha variable como central (Manzi y Silva, 2015).
} 
Así entonces, este esfuerzo investigativo apunta a avanzar en un conjunto de correlaciones y regresiones entre variables, frente a las cuales los individuos cristianos se comportarían de forma parecida, pero donde se cree que existen diferencias para católicos y evangélicos.

\section{VALORES Y DERECHOS HUMANOS}

Los valores son claves en el sistema de creencias, toda vez que - entre otras cosas - proporcionan — como señalan Schwartz y Bilsky (1990) criterios para evaluar a las personas, las situaciones, comportamientos concordantes con los valores que hacen sentir competentes y valorados socialmente. El actuar en contra de estos implica sentimientos de malestar creciente, esto hace que los valores actúen como motivadores de las conductas personales.

Según Schwartz y Bilsky (1990), los valores son: creencias (estructuras cognitivas ligadas a los afectos y sentimientos); refieren a metas deseables (igualdad, justicia, etc.); trascienden acciones y situaciones específicas (tienen pretensión de generalidad); sirven como estándares o criterios (guían la selección y la evaluación de acciones, políticas, etc.); están ordenados por importancia (poseen estructuras y jerarquías); y la importancia relativa del conjunto de valores relevantes orienta la acción de personas y grupos (todas las actitudes o conductas tienen distintas implicancias valorativas) (Schwartz y Bilsky, 1990; Correa, 2010).

La adopción e interiorización de valores se alcanza como fruto de un proceso constructivo del individuo que, en relación con otras personas embarcadas en el mismo proceso constructivo, trata de dar sentido a la realidad social que le rodea (García, Ramírez y Lima, 2008: 203).

En la construcción de valores se aprecia que los niños y jóvenes son agentes activos en la generación de los mismos, por lo que no se puede afirmar que exista una directa relación entre los valores que los padres desean que adquieran sus hijos y los que finalmente estos manifiesten. En esa medida, otras agencias socializadoras tales como la escuela, los pares, los medios de comunicación, las iglesias, juegan un papel complementario en el proceso formativo-valórico.

Estos valores ya instalados y operativos en los individuos y, por ende, en las relaciones que estos crean y en las cuales participan, se expresan en una solución que busca constituirlos en una modalidad de reconocimiento y validación común y compartida, que logre manifestarse más allá de las culturas y sus momentos históricos, y cuyas expresiones en este sentido se han plasmado en la Declaración Universal de los Derechos Humanos de 1948 (Aguilar, 2015). 


\section{EDUCACIÓN, CONTEXTO Y ESCUELA}

En torno a la educación se articulan los diversos discursos organizadores y de poder, que producen y reproducen, con el fin de generar una identidad, conservar sus adquisiciones, progresar en desarrollo, buscando la homogeneidad que los hace partícipes del mismo mundo y simultáneamente los hace diferentes y singulares en los espacios educativos.

Fundamental es mencionar que el espacio educativo más simbólico en nuestra sociedad actual es «la escuela», donde se aprenden, crean y reproducen los valores, derechos, deberes, las relaciones sociales o los distintos aspectos que se van generando o reconstruyendo por los distintos actores que participan en la misma. Además, se constituye en un espacio para las estrategias de los actores, donde se evidencia una aproximación de las desigualdades sociales, las convergencias de las relaciones o de las lecturas diferentes de la función de la escuela.

Por lo tanto, la educación impartida en las distintas instituciones forma parte de la realidad social, que es casi natural a la vida de todas las personas; no existe en la sociedad actual un cuestionamiento de por qué se debe ir y estar en la escuela, o en un establecimiento escolar, «a modo de mito, representa un conglomerado simbólico de significados, valores, aspiraciones y expectativas de comportamiento que operan en las formas de pensar, de querer $\mathrm{y}$ relacionarse los miembros de la sociedad» (Gimeno, 2000: 10).

En Chile, la sensibilidad por la formación en derechos humanos se expresa de un modo más concreto en lo que curricularmente se conoce como los Objetivos Fundamentales Transversales, que tienen un carácter comprensivo y general, cuyo logro se alcanza en el trabajo formativo del conjunto del currículum, o de subconjuntos de este que incluyen a más de un sector, subsector o especialidad. Es aquí donde se pueden identificar referencias directas a los derechos humanos y su correspondiente importancia en el proceso educativo.

\section{Planteamiento de las Iglesias Católica y EVANGÉLICA EN TORNO A LOS DERECHOS HUMANOS}

Los antecedentes actuales del comportamiento religioso de los chilenos se pueden recoger en la Encuesta Bicentenario PUC/Adimark Gfk (2014). Allí la población se distribuye del siguiente modo: católicos 59\%, evangélicos (iglesias protestantes en todas sus denominaciones) $16 \%$; otras religiones $3 \%$; sin religión o ateo $22 \%$.

Según Lalive D’Espinay (1968, en Mansilla, 2008), el protestantismo en Chile tendría dos momentos: uno a comienzos del siglo XX, en la formación de la nación chilena, y otro en la emergencia de las masas proletarias. En tal 
sentido, la estructura social actuaría como motor del aumento del pentecostalismo. Esto porque sería un refugio para las masas que migran del campo a la ciudad, están desamparadas de sus familias y ya no cuentan con el patrón. La comunidad pentecostal cumpliría una función de la hacienda: la integración del hombre a un grupo de relaciones personales directas y de dependencia. De allí la gran conversión al pentecostalismo en los sectores más pobres de la sociedad.

Según el autor (Lalive D’Espinay, 1968, en Mansilla, 2008), la Iglesia pentecostal brinda seguridad y permite sustraerse de peligros como los vicios o la mala conducta en el trabajo, que redundaría en despidos, pero, al mismo tiempo, es un lugar donde se rehúsa enfrentar y vencer aquello que amenaza a la sociedad. Por ello, el mismo autor sostiene que los movimientos pentecostales habrían sido presa de una anomia política. La fe se vive y practica en la comunidad religiosa y la comunidad es el entorno de mayor relevancia. En él se educa a los conversos en la conducta moral y en la vivencia del culto religioso. En una relación asimétrica con el pastor.

En el caso del catolicismo, el tema de los derechos humanos se puede reconocer con nitidez en los planteamientos del papa Juan XXIII, quien, en el año 1963, publicó la encíclica Pacem in Terris, en donde se lee con claridad la sensibilidad de la catolicidad mundial al respecto. En relación con Chile, si bien se asume lo indicado por Juan XXIII, está marcado fundamentalmente por el golpe de Estado de 1973; la Iglesia católica chilena se dedicó a prestar asistencia legal y social a quienes eran detenidos o torturados por el régimen militar. Con el retorno de la democracia (1990), los derechos humanos que más aparecen como defendidos por la Iglesia católica son aquellos relativos a la moral sexual y al derecho a la vida: aborto, eutanasia, control natural de la natalidad, homosexualidad, divorcio, entre otros (CECh, 2015).

\section{LA RELACIÓN ENTRE LAS MINORÍAS Y MAYORÍAS CREYENTES}

Las mayorías y minorías han sido objeto de estudio desde la política, la etnicidad y las religiones. Schermerhorn (1970) señaló que, en función de la representación numérica de los grupos religiosos y sus relaciones de poder, se conforman estratos dentro de la sociedad. Por ello, el tamaño relativo y el acceso directo al poder determinan si los grupos en una sociedad son dominantes o subordinados.

Cuando se aborda el fenómeno de la presencia de mayorías y minorías en grupos de diversa naturaleza, Coser (1961) sostiene que tanto en grupos religiosos como políticos se establecen identidades y un sentido de distinción en virtud de la pertenencia a un grupo, ya sea este minoritario o mayoritario. Malipaard y Phalet (2012) señalan que el contacto con grupos mayoritarios 
afecta la identidad de los grupos minoritarios. Sin embargo, en el contexto educativo no se da simplemente la lógica dicotómica de minoría v/s mayoría, o de relaciones de cordialidad $\mathrm{v} / \mathrm{s}$ relaciones de conflicto. Niens, O'Connor y Smith (2013) señalan que los jóvenes negocian sus identidades religiosas en una compleja red de interrelaciones entre la minoría de su comunidad de creencias religiosas y la cultura escolar dominante representada a través de las actitudes de los compañeros y del personal, de la ética escolar y de las prácticas, además de la educación religiosa.

Esta red de interrelaciones que establece el sujeto en cuanto a su posición de mayoría o minoría también debe tomar en cuenta que, a través de las generaciones, las identidades minoritarias tienden a diluir la fuerza con la que resisten la asimilación al grupo mayoritario (Platt, 2013). Las minorías en la segunda generación tendrían creencias y percepciones más cercanas a las de la mayoría.

\section{Metodología de LA INVESTigaCión}

\subsection{MODELO CONCEPTUAL}

El modelo de análisis contempla indagar en la relación entre ciertos factores del campo religioso de estudiantes y variables de derechos humanos. Para este estudio se consideran a los estudiantes cristianos, mayoritarios en la muestra original, y se estratifican por católicos y evangélicos. La búsqueda, primero de relaciones entre variables y luego influencia de variables consideradas independientes sobre otras dependientes, tiene como propósito configurar un comportamiento de los estudiantes en torno a aspectos significativos de derechos humanos.

A continuación, se presenta modelo teórico que se aplicó para estudiar el comportamiento de las variables:
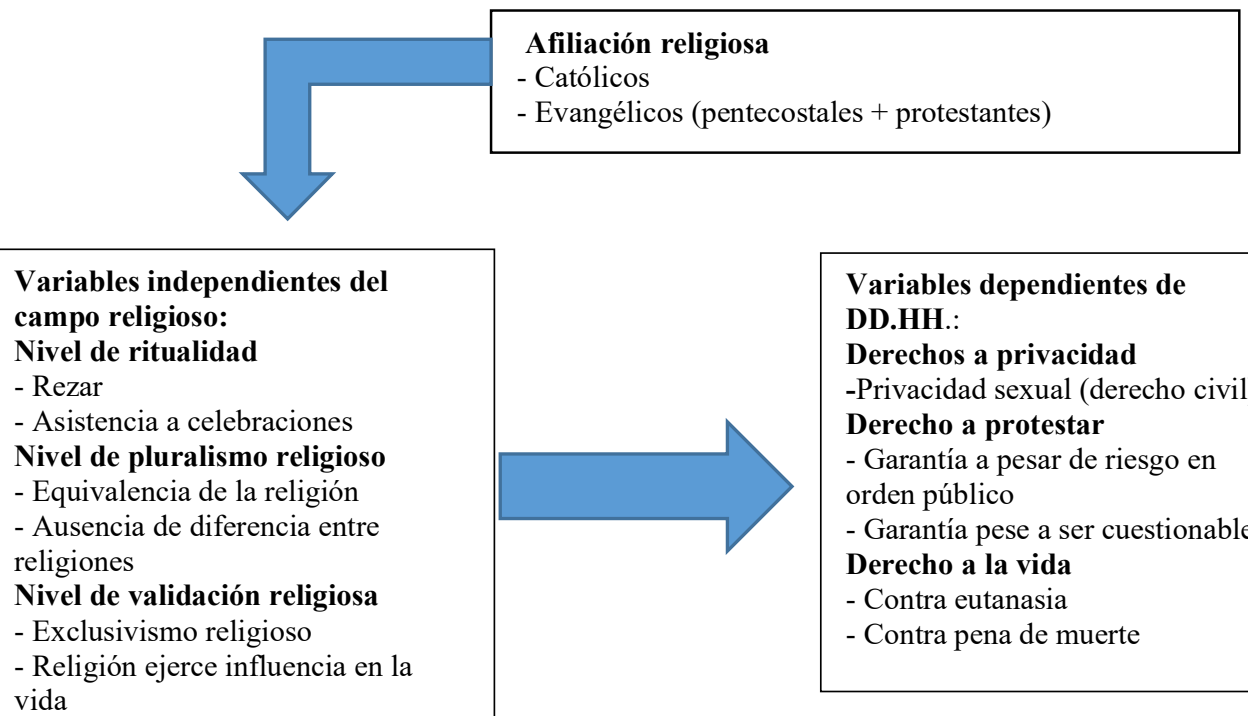

Variables dependientes de DD.HH.:

Derechos a privacidad

-Privacidad sexual (derecho civil)

Derecho a protestar

- Garantía a pesar de riesgo en orden público

- Garantía pese a ser cuestionable

Derecho a la vida

- Contra eutanasia

- Contra pena de muerte 
El tratamiento de los datos consistió en la realización de correlaciones y regresiones múltiples para cristianos, católicos y evangélicos.

\subsection{INTERPRETACIÓN DE RESULTADOS}

Las variables relacionadas con derechos humanos se agrupan en tres ámbitos: el derecho a la privacidad, ámbito fuertemente defendido de la incumbencia de los Estados, pero que en personas religiosas estaría influenciado por los marcos normativos de la religión; el derecho a protestar, fuertemente defendido en Estados democráticos, pero condicionado en ethos religiosos conservadores; y el derecho a la vida, específicamente en cuanto a poner término a ella a través de la eutanasia o de la pena de muerte, ambos casos criticados desde las religiones cristianas.

\subsection{CORRElaciones}

En la primera tabla de correlaciones para cristianos, observamos que las variables del campo religioso referidas a la ritualidad (asistencia a servicios religiosos y práctica de la oración) correlacionan significativa y negativamente con el derecho a la privacidad, es decir, si existe un elevado nivel de ritualidad en individuos, estos estarán menos de acuerdo con que se garantice que las personas practiquen en el ámbito de lo privado las actividades sexuales que estimen conveniente. Entonces, podemos esperar que jóvenes con mayor compromiso evidente con su religiosidad se planteen críticamente en el plano de la vida sexual, esperando límites al respecto.

El nivel de ritualidad también tiene una correlación significativa y ahora positiva con el derecho a la vida. Es decir, a mayor nivel de ritualidad, se estará más en contra de la despenalización de la eutanasia o de la promulgación de una pena de muerte. El nivel de ritualidad no correlaciona significativamente con el derecho a protestar, lo que puede explicarse a través de lo que sostienen las teorías de la secularización, que señalan que la religión se retira hacia el ámbito de lo privado y que en torno a lo púbico tiene menos vigor.

Cuando existe un alto pluralismo religioso, es decir, se considera una cierta equivalencia entre las religiones, existe una correlación significativa entre todas las variables independientes de derechos humanos. Es positiva para el caso del derecho a la privacidad, esto es, el individuo más pluralista se inclina por una mayor garantía de ejercer cualquier actividad sexual en el ámbito privado sin intervención del Estado; es negativa para el derecho a protestar y el derecho a la vida, o sea, personas más pluralistas cuestionan que los individuos puedan protestar poniendo en riesgo el orden público o si los motivos de las protestas son cuestionables. 
En cuanto a la validación religiosa, es decir, cómo la religión es fuertemente influyente en toda la vida de una persona y además se le considera como la única religión válida, se comporta significativamente y de manera negativa en relación con el derecho a la privacidad, esto es, no se puede hacer cualquier cosa que se estime conveniente, sino que debe ser escrutada por la mirada de la fe y del Estado. También correlaciona significativa y positivamente frente al derecho a la vida, lo que se entiende como el alto rechazo a la idea de la pena de muerte y a la eutanasia.

No se aprecia una correlación significativa en relación con el derecho a protestar. Es decir, «tu vida» puede estar fuertemente influida por «tu fe», pero no tiene ninguna implicancia, aunque seas minoría en la vida ciudadana, con el derecho a protestar.

\begin{tabular}{|l|c|c|c|}
\hline \multicolumn{4}{|c|}{ Tabla 1. Correlaciones: total cristianos (católicos y evangélicos) } \\
\hline & $\begin{array}{c}\text { Derecho a la } \\
\text { privacidad (civil) }\end{array}$ & $\begin{array}{c}\text { Derecho a protestar } \\
\text { (político) }\end{array}$ & $\begin{array}{c}\text { Derecho a la vida (contra } \\
\text { eutanasia y pena de } \\
\text { muerte) }\end{array}$ \\
\hline $\begin{array}{l}\text { Nivel de } \\
\text { ritualidad }\end{array}$ &,$- \mathbf{1 5 8}(* *)$ & 0,069 &, $\mathbf{2 9 5}(* *)$ \\
\hline $\begin{array}{l}\text { Nivel de } \\
\text { pluralismo } \\
\text { religioso }\end{array}$ &, $\mathbf{1 6 2}(* *)$ & $\mathbf{- , 1 2 0}(* *)$ & $\mathbf{- , 1 4 4 ( * * )}$ \\
\hline $\begin{array}{l}\text { Nivel de } \\
\text { validación } \\
\text { religiosa }\end{array}$ &,$- \mathbf{1 4 7}(* *)$ & 0,032 &, $\mathbf{2 6 1}(* *)$ \\
\hline \multicolumn{4}{|c|}{} \\
\hline
\end{tabular}

\subsection{DIFERENCIAS Y SIMILITUDES ENTRE CATÓLICOS Y EVANGÉLICOS}

En las tablas 2 y 3 observamos las mismas correlaciones, pero ahora separadas para católicos y evangélicos. Lo que se ve es que, para los católicos, el nivel de ritualidad no correlaciona con derechos del ámbito público o privado, sino más bien con un valor trascendental que es el derecho a la vida. Entonces, para católicos con alta asistencia a prácticas rituales y alta vida de oración, ponen énfasis en proteger a la vida, ya sea evitando la pena de muerte y/o repudiando la eutanasia.

En cambio, para los evangélicos, la ritualidad se correlaciona negativa y fuertemente con el ámbito de la sexualidad en lo privado. Entonces, para los evangélicos más practicantes, la sexualidad debe estar supeditada a lo planteado por su religión y no puede ejercerse al arbitrio de cualquier ciudadano.

El nivel de pluralismo religioso correlaciona negativamente con el derecho a protestar en católicos, es decir, católicos más pluralistas no están de 
acuerdo con protestas que no garanticen el orden público o sean cuestionables. En el caso de evangélicos más pluralistas, esto es, que ven su religión como equivalente a otras, estarán más de acuerdo con que se garantice la privacidad de las personas y puedan practicar en lo privado la actividad sexual que estimen conveniente.

En cuanto al nivel de validación religiosa, en católicos correlaciona significativa y negativamente con el derecho a la privacidad y positivamente con el derecho a la vida. Para el caso de evangélicos, solo correlaciona significativa y positivamente con el derecho a la vida.

Tabla 2. Correlaciones católicos y evangélicos

\begin{tabular}{|l|r|r|r|}
\hline \multicolumn{4}{|c|}{ Correlaciones: católicos } \\
\hline & $\begin{array}{l}\text { Derecho a la } \\
\text { privacidad } \\
\text { (civil) }\end{array}$ & $\begin{array}{c}\text { Derecho a } \\
\text { protestar } \\
\text { (politico) }\end{array}$ & $\begin{array}{c}\text { Derecho a la } \\
\text { vida (contra } \\
\text { eutanasia y } \\
\text { pena de } \\
\text { muerte) }\end{array}$ \\
\hline Nivel de Ritualidad & $-0,039$ & $-0,007$ &, $212\left(^{* *}\right)$ \\
\hline $\begin{array}{l}\text { Nivel de Pluralismo } \\
\text { religioso }\end{array}$ & 0,051 &,$- 096\left(^{* *}\right)$ & $-0,077$ \\
\hline $\begin{array}{l}\text { Nivel de Validación } \\
\text { religiosa }\end{array}$ &,$- 117\left(^{*}\right)$ & $-0,048$ &, $190\left(^{* *}\right)$ \\
\hline
\end{tabular}

* Correlation is significant at the 0.05 level (2-tailed).

\begin{tabular}{|l|r|r|r|}
\hline \multicolumn{4}{|c|}{ Correlaciones: evangélicos } \\
\hline & $\begin{array}{c}\text { Derecho a la } \\
\text { privacidad } \\
\text { (civil) }\end{array}$ & $\begin{array}{c}\text { Derecho a } \\
\text { protestar } \\
\text { (politico) }\end{array}$ & $\begin{array}{c}\text { Derecho a la } \\
\text { vida (contra } \\
\text { eutanasia y } \\
\text { pena de } \\
\text { muerte) }\end{array}$ \\
\hline Nivel de Ritualidad &,$- 304(* *)$ & 0,124 & 0,146 \\
\hline $\begin{array}{l}\text { Nivel de Pluralismo } \\
\text { religioso }\end{array}$ &, $339(* *)$ & $-0,061$ & $-0,111$ \\
\hline $\begin{array}{l}\text { Nivel de Validación } \\
\text { religiosa }\end{array}$ & $-0,103$ & 0,153 &, $225(* *)$ \\
\hline
\end{tabular}

** Correlation is significant at the 0.01 level (2-tailed).

** Correlation is significant at the 0.01 level (2-tailed).

\subsection{REGRESIONES}

\section{Cristianos}

En el total de cristianos, el modelo para el derecho a privacidad y derecho a protestar recibe influencia significativa solo del nivel de pluralismo religioso, y los modelos completos explican poco del cambio de la variable dependiente ( $4 \%$ y $1 \%$, respectivamente). Esto puede interpretarse como que las variables de práctica y validación religiosa no ejercen influencia en aspectos relativos al comportamiento de los individuos en la esfera pública o privada. Sin embargo, el modelo que mide el efecto de las variables independientes del campo religioso sobre la variable dependiente derecho a la vida sí es significativo para las tres variables y explica el $12 \%$ del cambio en la variable dependiente. Es decir, en el modelo es más sensible el derecho a la vida.

\begin{tabular}{|l|c|c|c|}
\hline \multicolumn{4}{|c|}{ Tabla 3. Regresiones lineales, total cristianos (católicos y evangélicos) } \\
\hline & $\begin{array}{c}\text { Derecho a la } \\
\text { privacidad (civil) }\end{array}$ & $\begin{array}{c}\text { Derecho a } \\
\text { protestar } \\
\text { (político) }\end{array}$ & $\begin{array}{c}\text { Derecho a la vida } \\
\text { (contra eutanasia y pena } \\
\text { de muerte) }\end{array}$ \\
\hline Nivel de ritualidad & 0,06 & 0,232 & o \\
\hline
\end{tabular}



diferencias en estudiantes secundarios chilenos

\begin{tabular}{|l|c|c|c|}
$\begin{array}{l}\text { Nivel de pluralismo } \\
\text { religioso }\end{array}$ & $\mathbf{0}$ & $\mathbf{0 , 0 0 2}$ & $\mathbf{0}$ \\
\hline $\begin{array}{l}\text { Nivel de validación } \\
\text { religiosa }\end{array}$ & 0,043 & 0,995 & $\mathbf{0 , 0 0 1}$ \\
\hline Adjusted R Square & $\mathbf{0 , 0 4 9}$ & $\mathbf{0 , 0 1 7}$ & $\mathbf{0 , 1 2}$ \\
\hline Sig & $\mathbf{0 0 0 ( a )}$ & $\mathbf{, 0 0 6 ( a )}$ & $\mathbf{0 0 0 ( a )}$ \\
\hline
\end{tabular}

\section{Católicos y evangélicos}

Los modelos para católicos también concentran su significancia en el derecho a la vida, pero explican menos el cambio en la variable dependiente (6\%). Sin embargo, en el caso de evangélicos, el nivel de ritualidad y pluralismo influencia el derecho a la privacidad, en un modelo que explica el $15 \%$ de la variable dependiente. Para evangélicos también es sensible el derecho a la vida al cambio en todas las variables del campo religioso, en un modelo que explica el $12 \%$ del cambio en la variable dependiente derecho a la vida.

Tabla 4.

\begin{tabular}{|l|r|r|r|}
\hline \multicolumn{4}{|c|}{ Regresiones lineales católicos } \\
\hline & $\begin{array}{c}\text { Derecho a la } \\
\text { privacidad } \\
\text { (civil) }\end{array}$ & $\begin{array}{c}\text { Derecho a } \\
\text { protestar } \\
\text { (politico) }\end{array}$ & $\begin{array}{l}\text { Derecho a la } \\
\text { vida (contra } \\
\text { eutanasia y } \\
\text { pena de } \\
\text { muerte) }\end{array}$ \\
\hline Nivel de Ritualidad & 0,815 & 0,624 & $\mathbf{0 , 0 0 2}$ \\
\hline $\begin{array}{l}\text { Nivel de Pluralismo } \\
\text { religioso }\end{array}$ & 0,169 & 0,02 & $\mathbf{0 , 0 0 8}$ \\
\hline $\begin{array}{l}\text { Nivel de Validación } \\
\text { religiosa }\end{array}$ & 0,027 & 0,464 & $\mathbf{0 , 0 1 3}$ \\
\hline Adjusted R Square & 0,009 & 0,008 & $\mathbf{0 , 0 6 6}$ \\
\hline Sig &, $076(a)$ &, $098(a)$ &, $\mathbf{0 0 0}(\mathbf{a})$ \\
\hline
\end{tabular}

\begin{tabular}{|l|r|r|r|}
\hline \multicolumn{4}{|c|}{ Regresiones lineales evangélicos } \\
\hline & $\begin{array}{c}\text { Derecho a la } \\
\text { privacidad } \\
\text { (civil) }\end{array}$ & $\begin{array}{c}\text { Derecho a } \\
\text { protestar } \\
\text { (politico) }\end{array}$ & $\begin{array}{l}\text { Derecho a la } \\
\text { vida (contra } \\
\text { eutanasia y } \\
\text { pena de } \\
\text { muerte) }\end{array}$ \\
\hline Nivel de Ritualidad & $\mathbf{0 , 0 2}$ & 0,619 & 0 \\
\hline $\begin{array}{l}\text { Nivel de Pluralismo } \\
\text { religioso }\end{array}$ & $\mathbf{0 , 0 0 1}$ & 0,51 & 0 \\
\hline $\begin{array}{l}\text { Nivel de Validación } \\
\text { religiosa }\end{array}$ & 0,859 & 0,203 & $\mathbf{0 , 0 0 1}$ \\
\hline Adjusted R Square & $\mathbf{0 , 1 5 1}$ & $\mathbf{0 , 0 0 8}$ & $\mathbf{0 , 1 2}$ \\
\hline Sig & $\mathbf{0 0 0 ( a )}$ &, $269(a)$ &, $000(a)$ \\
\hline
\end{tabular}

\section{CONCLUSIONES}

Pese a que los datos recogidos indican débiles relaciones entre las variables independientes y dependientes, ya sea si consideramos a los creyentes cristianos como un todo o cuando los casos se analizan por separado en católicos y evangélicos, esto parecería normal al considerar el cúmulo de otras variables no observadas que intervienen en el fenómeno.

Sin embargo, las tendencias observadas dan cuenta de un comportamiento que, si bien es parecido entre católicos y evangélicos, presenta ciertas diferencias que pueden interpretarse sobre la base de los elementos teóricos aquí planteados, que aluden a dinámicas de relación de mayoríaminoría y de prácticas rituales, entre otros. 
Como se señala en este documento, los valores nos proporcionan criterios para evaluar a las personas, las situaciones y a nosotros mismos. En esta investigación, estudiantes cristianos evaluaron ciertas situaciones que se plasman en derechos humanos y en esta evaluación intervinieron sus creencias y su posible comportamiento frente a dichas situaciones (Schwartz y Bilsky, 1990). Como señalan Schwartz y Bilsky (1990), los valores poseen estructuras de jerarquía $y$, en ese sentido, es comprensible que dos tipos de religiones que siguen estudiantes de educación secundaria, pese a su misma raíz cristiana, impliquen valoraciones diferentes frente a determinados derechos humanos.

La fuerza que pueden ejercer las agencias socializadoras donde la religión juega un rol de importancia, también intervienen en las posiciones de los estudiantes frente a determinados derechos humanos, por ello, si en la familia o en la escuela se forman estructuras cognitivas de mayor exclusividad de la religión, apoyada en una práctica religiosa frecuente, podemos visualizar que los resultados son coherentes con una oposición a la pena de muerte y a la eutanasia y donde la privacidad sexual sin intervención del Estado es rechazada.

Un elemento propio de la modernidad es el pluralismo religioso, entendido como la aceptación de la presencia de otras religiones en la sociedad, sin que ello lleve a conflicto (Berger y Luchmann, 1995). Si asumimos que el pluralismo religioso va introduciéndose en las sociedades más modernas, se podría hipotetizar que aquellas serán las opiniones generalizadas en las sociedades a futuro.

En estos contextos, como se ha indicado en el análisis de la investigación, se van generando identidades que hacen que los estudiantes se sientan partícipes del mismo mundo, aunque son ellos mismos singulares. Esta importancia de la agencia escuela en la formación de valores en los estudiantes es radical cuando la agencia primaria, que es la familia, ya no es tan potente, como sucede en la adolescencia. Si la agencia escuela está cohesionada con la identidad religiosa, puede efectivamente ejercer influencia en los «valores, aspiraciones y expectativas de comportamiento que operan en las formas de pensar, de querer y de relacionarse de los miembros de la sociedad») (Gimeno, 2000: 10). Por ello, queda para una posterior investigación reconocer cuánto es lo que influye la escuela en la opinión del estudiante según su tipo de religión cristiana. Por lo pronto, se puede afirmar que, en los matices que se encuentran en los resultados entre católicos y evangélicos, también podría estar ejerciendo una influencia significativa la escuela de procedencia del alumno, ya sea para acentuar una religiosidad de exclusividad y de práctica religiosa frecuente o para intencionar la diversidad cultural mediante un pluralismo religioso.

Dadas las sensibilidades con que se valoran los derechos humanos en la actualidad, desde lo religioso para los católicos, nos permite comprender que los estudiantes se inclinen a valorar más derechos humanos orientados a un mayor rechazo a la pena de muerte y a la eutanasia, así como que la sexualidad, en el contexto de privacidad, debiera estar regulada por el Estado. Sin embargo, 
no se manifiestan con relación a derechos civiles, como el derecho a protestar o similares.

Cuando el católico es más pluralista, dejan de tener importancia las temáticas de la sexualidad y del derecho a la vida y toma fuerza el derecho a protestar, pero sin poner en peligro el orden público y donde estas protestas deben basarse en temas no cuestionables.

Por otra parte, los evangélicos son una religión que está en Chile desde principios del siglo XX y, aunque va en aumento, siempre ha sido minoritaria en el país. Los estudiantes evangélicos forman parte de la segunda o tercera generación de evangélicos, es decir, en su mayoría no son convertidos a la fe evangélica, sin embargo, su linaje creyente está rodeado de simbolismos y convicciones que en ocasiones tienen mayor fuerza que en el caso del catolicismo. No obstante, y por las características de la religión - minoritaria y sin mayor poder en la sociedad (Schermerhorn, 1970) - , ha sido presa de una «anomia política» (D’Espinay, 1968, en Mansilla, 2008). Por ello, son coherentes los resultados en que estudiantes evangélicos con mayor práctica religiosa y que consideran su religión como la exclusiva y válida apoyen que el Estado intervenga en las relaciones sexuales en contexto de privacidad y que rechacen la pena de muerte y la eutanasia. Sin embargo, en los temas públicos de derecho a protestar no existen resultados significativos, por lo que podríamos afirmar que no existe relación con estos derechos públicos, lo que coincide con la llamada «anomia política».

Los estudiantes católicos están más significativamente inclinados a rechazar aquello que atente contra el derecho a la vida (eutanasia y pena de muerte), y no a la privacidad sexual ni al derecho a protestar. Sin embargo, en evangélicos es relevante que el Estado participe de la regulación de la práctica sexual en contexto privado, así como rechazan la eutanasia y la pena de muerte.

Como se puede apreciar, existen, aunque no excesivamente notorias, diferencias entre estudiantes católicos y evangélicos, miembros de la raíz cristiana de la sociedad chilena. Estas diferencias pueden explicarse por cómo se han configurado ambas religiones en la historia de nuestro país en lo concerniente a valores, derechos humanos, si son mayoría o minoría y en contexto de escuela. Este último punto constituye la apertura para una nueva investigación que agregue como variable la identidad de la escuela, para conocer si las diferentes identidades de ellas juegan un papel entre la religión de los estudiantes y su posición frente a los derechos humanos.

RECIBIDO: 26 DE DICIEMBRE DE 2018

ACEPTADO: 19 DE JUNIO DE 2019 


\section{BibLIOGRAFÍA}

Aguilar, M. (2015). Las tres generaciones de los Derechos Humanos. Disponible en:

http://www.juridicas.unam.mx/publica/librev/rev/derhum/cont/30/pr/pr2 $0 . p d f$

Berger, P. y LuckMAnN, T. (1997). Modernidad, pluralismo y crisis de sentido: La orientación del hombre moderno. Barcelona. Paidós.

CORREA, P. (2010). «Imágenes de Dios y su relación con el sistema valorativo: el caso de los jóvenes estudiantes de educación superior de la región metropolitana». Tesis de grado para optar al título de sociólogo. Universidad de Chile, Santiago.

CONFERENCIA EPISCOPAL DE CHILE. (2015). El derecho humano a la vida, a una vida digna para toda persona. Disponible en:

$\mathrm{http}: / /$ documentos.iglesia.cl/conf/doc_pdf.php?mod=documentos_sini\&i $\mathrm{d}=4356$

Coser, L. A. (1961). Las funciones del conflicto social. México, D.F.: Fondo de Cultura Económica.

ENCUESTA BICENTENARIO PUC-ADIMARK. (2014). Disponible en: http://encuestabicentenario.uc.cl/wp-content/uploads/2014/11/UCAdimark-2014.pdf

GARCíA, M., RAmírez, G. y LimA, A. (2008). «La construcción de valores en la familia». En M. J. RodRIGO y J. PALACIOS (Coords.), Familia y desarrollo humano. Madrid: Alianza.

GiMENo, J. (2000). La educación obligatoria: su sentido educativo y social. Madrid: Morata.

MAliePAard, M. y Phalet, K. (2012). «Social Integration and Religious Identity Expression among Dutch Muslims The Role of Minority and Majority Group Contact». Social Psychology Quarterly, 75: 131-148.

MANSILla AGÜERO, M. (2008). «Pluralismo, subjetivización y mundanización. El impacto de la secularización en el neopentecostalismo chileno». Revista Latinoamericana, POLIS, 19.

MANZI, J. y SiLVA J. (2014). «La religión en el espacio público: Diversas percepciones entre jóvenes chilenos de la separación entre Iglesia y Estado». Presentación realizada en 2nd Conference Religion and Human Rights (RHR), 10-13 de diciembre de 2014. Würzburg, Alemania. . (2015). «Societal functions of religion and their relationships with human rights support in Chile». Presentación realizada en 3rd Conference Religion and Human Rights (RHR), 9-12 de diciembre de 2015, Zagreb, Croacia. 
Mieke, M. y Phalet, K. (2012). «Social Integration and Religious Identity Expression among Dutch Muslims: The role of minority and majority Group contact». Social Psychology Quarterly, 2(9): 131-148.

MiRES, F. (2006). La colonización de las almas: misión y conquista en Hispanoamérica. Buenos Aires: Libros de la Araucaria.

NiEns, U., O’CONNOR, U. y SMITH, A. (2013). «Citizenship education in divided societes: Teachers' perspective in Northern Ireland». Citizenchip Studies, 17(1): 128-141.

Orellana, L. (2008). El fuego y la nieve: historia del Movimiento Pentecostal en Chile 1909-1932. Madrid: CEEP.

Platt, L. (2014). «Is there Assimilation in Minority Groups' National, Ethnic, and Religious Identity?». Ethnic and Racial Studies, 37: 46-70.

SCHERMERHORN, R. (1970). Comparative Ethnic Relations: A Framework for Theory and Research. Nueva York: Random House.

SCHWARTZ, S. y BILSKY, W. (1990). «Toward a Theory of the Universal Content and Structure of Values: Extension and Cross- Cultural Replications». Journal of Personality and Social Psychology, 58(5): 878-891.

Ulrike, N., Mawhinney, A., Richardson, N. y ChibA, Y. (2013). «Acculturation and religion in schools: the views of young people from minority belief backgrounds». British Educational Research Journal, 39(5): 907-924.

ZIEBERTZ, H.-G. y REINDL, M. (2012). «Religious socialisation and values as predictors of human rights attitudes: An empirical study among Christian and Muslim adolescent in Germany». En J. A. VAN DER VEN y H.-G. ZIEBERTZ (Eds.), Tensions within and betwen religious and human rights (pp. 223-247). Leiden: Brill. 